\title{
A Pleasant Reading of GARCH Model
}

\section{Viviane Y. Naïmy*}

Faculty of Business Administration \& Economics, Notre Dame University- Louaize, Lebanon, USA

Measuring and forecasting the size of the errors in any model is a must. The ARCH/GARCH, which stand for autoregressive conditional heteroskedasticity and generalized autoregressive conditional heteroskedasticity, treat heteroskedasticity as a variance to be modeled when the variances of the error terms are not equal ${ }^{1}$. The ARCH/ GARCH corrects the least squares flaws and predict the variance of each error term. The main concern, even after the development of the "robust standard error" is the variance of the error terms and what makes them large. This problem is often found in investment where the dependent variable is the return on an asset and the variance of the return represents the risk level of those returns. In such time series analysis of return the heteroskedasticity constitutes a serious handicap. In risky periods, the expected value of the magnitude of error terms is high and during normal periods, it is not. Therefore, there is a degree of autocorrelation in the riskiness of returns. The goal of ARCH and GARCH is to provide a volatility measure in order to facilitate financial decisions.

Assuming that $r_{\mathrm{t}}$ is the return on an asset (or portfolio). The mean value $m$ and the variance $h$ are defined relative to a past data set. Therefore, the current return $r$ is equal to the mean value of $r$ plus the standard deviation of $r$ times the error term for the present period. Forecasting the mean and variance based on past data is only possible through ARCH model. The equal weights hypothesis together with the zero weights for observations exceeding one month are implausible because recent events will highly affect the expectation of the variance. The ARCH model as suggested by Engle [1] solved the problem by estimating the parameters corresponding to the best weights in forecasting the variance. The GARCH, introduced by Bollerslev [2], defined a practical generalization of the $\mathrm{ARCH}$; it allocates declining weights for past squared residuals which never reach zero. Accordingly, the variance predictor for the next period is a weighted average of the long run average variance, of the predicted variance for the current period, and the new information of the period which is the recent squared residual. As defined by Engle [3], when $h_{t}$ is the variance of the residuals of a regression $r_{t}=m+\left.\sqrt{ } h_{t}\right|_{\mathrm{t}}$, the variance of $\left.\right|_{\mathrm{t}}$ is one. The GARCH for variance is then:

$$
h_{\mathrm{t}+1}=\omega+\alpha\left(r_{\mathrm{t}}-m_{\mathrm{t}}\right)^{2}+\beta h t=\omega+\alpha h_{\mathrm{t}} \mid \mathrm{t}^{2}+\beta h t
$$

The constants $\omega, \alpha$ and $\beta$ are estimated and the previous forecast $h$ and residuals are known. The weights are $1-\alpha-\beta, \beta, \alpha$, and the long run average variance is $\sqrt{ }(\omega /(1-\alpha-\beta)$ provided $\alpha+\beta<1$ and $\omega>0, \alpha>0$, and $\beta>0$. This model is called GARCH $(1,1)^{2}$.

The estimation of GARCH equation is simple and straightforward when software like SAS, RATS, Matlab and many others use the Maximum Likelihood based on the variable $r_{\mathrm{t}}$ by substituting $h_{\mathrm{t}}$ for the $\sigma^{2}$. In order to check whether the true variance differs from the one specified by the analyst, a simple test can be done by constructing the sets of $\left\{\left.\right|_{t}\right\}$ and measuring their mean and variance supposed to be constant. Ljung Box Test ${ }^{3}$ [4] for autocorrelation in the squares is able to detect the model significance accuracy.

\section{References}

1. Engle, Robert F (1982) Autoregressive Conditional Heteroscedasticity with Estimates of the Variance of United Kingdom Inflation. Econometrica 50: 9871007.

2. Bollerslev, Tim (1986) Generalized Autoregressive Conditional Heteroskedasticity. Journal of Econometrics 31: 307-327.

3. Engle, Robert $F$ (2001) GARCH 101: The Use of ARCH/GARCH Models in Applied Econometrics. The Journal of Economic Perspectives 15: 157-168.

4. Ljung GM, Box GEP (1978) On a measure of Lack of Fit in a Time Series Models. Biometrica 65: 297-303.
${ }^{1}$ The basic version of the least squares model assumes that the expected value of all error terms when squared is the same at any given point.

${ }^{2}$ The first number refers to how many autoregressive lags appear in the equation, while the second number refers to how many moving average lags are specified (number of GARCH terms).

${ }^{3}$ This test is used with 15 lagged autocorrelations.
*Corresponding author: Viviane Y. Naïmy, Faculty of Business Administration \& Economics, Notre Dame University-Louaize, Lebanon, USA E-mail: vnaimy@ndu.edu.lb

Received January 21, 2013; Accepted January 22, 2013; Published January 23 2013

Citation: Naïmy VY (2013) A Pleasant Reading of GARCH Model. J Bus \& Fin Aff 2: e132. doi:10.4172/2167-0234.1000e132

Copyright: @ 2013 Naïmy VY. This is an open-access article distributed under the terms of the Creative Commons Attribution License, which permits unrestricted use, distribution, and reproduction in any medium, provided the original author and source are credited. 